Research Article

\title{
A Novel Passive Circuit Emulator for a Current-Controlled Memristor
}

\author{
Leonardo Barboni \\ Department of Electronica, Instituto de Ingenieria Electrica-FING, Julio Herrera y Reissig 565 11300, Montevideo, Uruguay \\ Correspondence should be addressed to Leonardo Barboni; lbarboni@fing.edu.uy
}

Received 2 February 2021; Revised 23 March 2021; Accepted 16 April 2021; Published 22 April 2021

Academic Editor: Luigi Di Benedetto

Copyright (C) 2021 Leonardo Barboni. This is an open access article distributed under the Creative Commons Attribution License, which permits unrestricted use, distribution, and reproduction in any medium, provided the original work is properly cited.

\begin{abstract}
A memristor is an electrical element, which has been conjectured in 1971 to complete the lumped circuit theory. Currently, researchers use memristor emulators through diodes, inductors, and other passive (or active) elements to study circuits with possible attractors, chaos, and ways of implementing nonlinear transformations for low-voltage novel computing paradigms. However, to date, such passive memristor emulators have been voltage-controlled. In this study, a novel circuit realization of a passive current-controlled passive inductorless emulator is established. It overcomes the lack of passive current-controlled memristor commercial devices, and it can be used as part of more sophisticated circuits. Moreover, it covers a gap in the state of the art because, currently, only passive circuit voltage-controlled memristor emulators and active current-controlled emulators have been developed and used. The emulator only uses two diodes, two resistors, and one capacitance and is passive. The formal theory and simulations validate the proposed circuit, and experimental measurements were performed. The parameter conditions of numerical simulations and experiments are consistent. Simulations were performed with an input current amplitude of $15 \mathrm{~mA}$ and frequencies of up to $3 \mathrm{kHz}$ and measurements were carried out with an input current amplitude of $0.74 \mathrm{~mA}$ and frequency of $1.5 \mathrm{kHz}$ in order to compare with the state of the art.
\end{abstract}

\section{Introduction}

A memristor is an electrical two-terminal passive nonlinear resistance element that exhibits a well-known pinched hysteresis loop at the origin of the voltage-current plane when any bipolar periodic zero-mean excitatory voltage or current of any value is applied across it. However, there are disagreements regarding whether a memristor can be considered a fundamental element and whether its dynamic is purely electromagnetic, as originally conjectured, or if there are other mechanisms involved such as ionic transport. Despite the controversy surrounding the technological realization, the amount of research into the properties of the pinched hysteresis loop continues to increase. Irrespective of whether the memristor is implemented by emulating its behavior through circuitry composed of other active or passive components, new studies continue to generate and sustain optimistic expectations in the scientific community about the use and advantages of the memristor. The research interest in the memristor is motivated by its promising potential for building novel integrated circuits and computing systems, as has been proposed in [1-7]. Memristors allow the memory and time-varying processing of information through nonlinear transformations in a unique passive element.

There is neither implementation nor a proof-of-concept for a passive current-controlled memristor system-on-chip but yes for an active current-controlled memristor [8]. With an increasing need to better understand a pinched hysteresis attractor, researchers implement memristor emulators through diodes and other passive (or active) elements. Such emulators allow studying (theoretically and numerically) possible attractors and ways of implementing nonlinear transformations for novel computing paradigms. The first voltage-controlled memristor emulator has been proposed in [9] and was based on a diode bridge with a parallel $R-C$ filter as a load. Other studies [10-16] used voltage-controlled memristor emulators as vital building blocks of other circuits for the in-depth study of their bifurcations and chaotic behaviors. 
To summarize, (a) background: to the best of our knowledge and after searching in database providers, we have concluded that a passive and current-controlled memristor emulator has not been published previously (only active current-controlled memristor [8]) (although there are a lot of voltage-controlled memristor emulators) and (b) motivation: design a passive and current-controlled memristor emulator for neuromorphic computing and as a candidate for mimicking synaptic functions and inductorless emulator that could be easily integrated into CMOS technology. It is similar in spirit to some circuits in the literature, which combine a rectifier with a LC low pass filter and not an $\mathrm{RC}$ as it is here. This memristor enables its utilization in programmable circuits and systems controlled by digital pulses.

Memristors exhibit three characteristics for any bipolar periodic signal excitation: (i) there is a pinched hysteresis loop in the voltage-current plane, (ii) the area of the hysteresis loop decreases and shrinks to a single-valued $V-I$ function when the signal excitation frequency tends to infinity, and (iii) for voltage-controlled generalized memristive time-invariant systems, the following equations apply [1-7]:

$$
\left\{\begin{array}{l}
i_{m}=G\left(\mathbf{x}, V_{m}\right) V_{m} \text { and } G(\mathbf{x}, 0) \neq \infty \forall \mathbf{x}, \\
\frac{\mathrm{d} \mathbf{x}}{\mathrm{d} t}=f\left(\mathbf{x}, V_{m}\right) \mathbf{x} \text { represents the inner state variables, }
\end{array}\right.
$$

where $i_{m}$ is the current across the memristor, $V_{m}$ is the voltage across the terminals, $G\left(\mathbf{x}, V_{m}\right)$ is bounded, and $f\left(\mathbf{x}, i_{m}\right)$ is the equation of state, which must be also bounded to guarantee the existence of a solution $\mathbf{x}(t)$. The area of the lobes, shapes, and orientation of the hysteresis loop evolves with frequency. All of the abovementioned references developed voltage-controlled memristor emulators (i.e., equations such as equation (1)). However, in this study, we implement the first-ever built passive current-controlled memristor emulator for which the following equations apply:

$$
\left\{\begin{array}{l}
V_{m}=R\left(\mathbf{x}, i_{m}\right) i_{m} \text { and } R(\mathbf{x}, 0) \neq \infty \forall \mathbf{x}, \\
\frac{\mathrm{d} \mathbf{x}}{\mathrm{d} t}=f\left(\mathbf{x}, i_{m}\right) \mathbf{x} \text { represents the inner state variables. }
\end{array}\right.
$$

\section{Proposed Current-Controlled Memristor}

Novel current-controlled memristor emulators have been introduced recently in $[8,17,18]$; however, all of them are active (it means that a voltage power supply is needed). In this work, the first passive circuit realization of a currentcontrolled emulator is established in Figure 1. It uses two diodes, two resistors, and one capacitor.

We construct the equations by beginning with the Shockley diode equation (both diodes are equal and without considering high-frequency effects that produce unwanted dynamic effects):

$$
\begin{aligned}
& i_{D_{1}}=I_{s}\left(e^{2 \alpha V_{j D_{1}}}-1\right), \\
& i_{D_{2}}=I_{s}\left(e^{2 \alpha V_{j D_{2}}}-1\right),
\end{aligned}
$$

where $2 \alpha=1 / n V_{T}$ and $I_{s}$ denote the reverse saturation current, $n$ is the emission coefficient, $V_{T}$ is the thermal voltage, and $V_{j D_{1}}$ and $V_{j D_{2}}$ are the junction diode voltages. If we consider their series parasitic resistance $R_{p}$, the diode voltages become

$$
V_{D_{1}}=R_{p} i_{D_{1}}+\frac{1}{2 \alpha} \ln \left(1+\frac{i_{D_{1}}}{I_{s}}\right) \text { and } V_{D_{2}}=R_{p} i_{D_{2}}+\frac{1}{2 \alpha} \ln \left(1+\frac{i_{D_{2}}}{I_{s}}\right) \text {. }
$$

Therefore,

$$
i_{D_{1}}=I_{s}\left(e^{2 \alpha\left(V_{D_{1}}-R_{p} I_{D_{1}}\right)}-1\right) \text { and } i_{D_{2}}=I_{s}\left(e^{2 \alpha\left(V_{D_{2}}-R_{p} I_{D_{2}}\right)}-1\right) .
$$

According to the voltage drop,

$$
\begin{gathered}
V_{m}=R i_{1}+V_{D_{1}} \text { and } V_{m}=R i_{2}-V_{D_{2}}, \\
-V_{c}=V_{D_{1}}+V_{D_{2}} .
\end{gathered}
$$

The current $i_{m}$ corresponds to $i_{m}=i_{D_{1}}-i_{D_{2}}$. Using equation (5),

$$
\left.i_{m}=I_{s} e^{\alpha\left(V_{D_{1}}+V_{D_{2}}-R_{p}\left(i_{D_{1}}+i_{D_{2}}\right)\right.}\right)\left(e^{\alpha\left(V_{D_{1}}-V_{D_{2}}-R_{p}\left(i_{D_{1}}-i_{D_{2}}\right)\right)}-e^{-\alpha\left(V_{D_{1}}-V_{D_{2}}-R_{p}\left(i_{D_{1}}-i_{D_{2}}\right)\right)}\right) .
$$

Using equation (7), it becomes convenient to express

$$
i_{m}=2 I_{s} e^{-\alpha V_{c}} e^{-\alpha R_{p}\left(i_{D_{1}}+i_{D_{2}}\right)} \sinh \left(\alpha\left(V_{D_{1}}-V_{D_{2}}-R_{p} i_{m}\right)\right) .
$$

However, from equation (6),

$$
2 V_{m}=R i_{m}+V_{D_{1}}-V_{D_{2}} .
$$

Then, the $\left\{V_{m}-i_{m}\right\}$ relation is provided, 


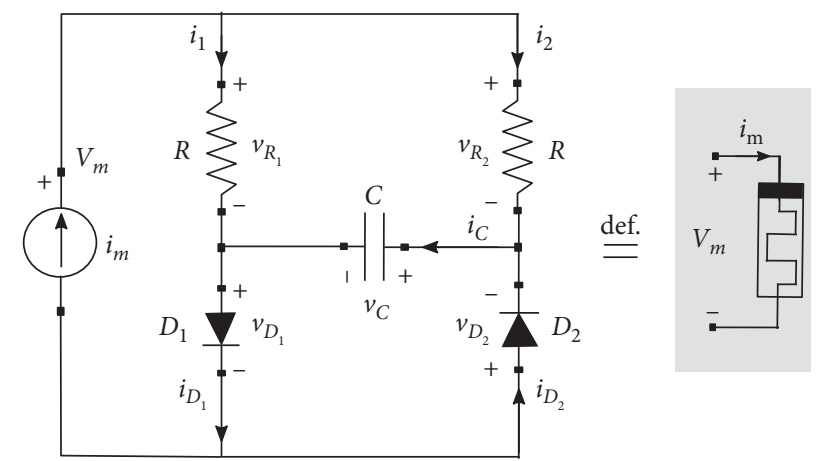

(a)

(b)

FIgURe 1: (a) Proposed current-controlled memristor circuit emulator and (b) generalized symbol of the memristor device.

$$
V_{m}=\frac{1}{2} R i_{m}+\frac{1}{2 \alpha} \sinh ^{-1}\left(\frac{i_{m}}{2 I_{s}} e^{\alpha V_{c}+\alpha R_{p}\left(i_{D_{1}}+i_{D_{2}}\right)}\right)+\frac{1}{2} R_{p} i_{m} .
$$

Because $i_{c}=i_{D_{1}}-i_{m}+i_{2}$ and $i_{c}=i_{D_{2}}+i_{2}$, we obtain $i_{D_{1}}+i_{D_{2}}=2 i_{c}+i_{m}-2 i_{2}$ and then,

$$
V_{m}=\frac{1}{2} R i_{m}+\frac{1}{2 \alpha} \sinh ^{-1}\left(\frac{i_{m}}{2 I_{s}} e^{\alpha V_{c}+\alpha R_{p}\left(2 i_{c}+i_{m}-2 i_{2}\right)}\right)+\frac{1}{2} R_{p} i_{m}
$$

Next, we focus on the $i_{2}$ expression. By taking $V_{m}=$ $V_{D_{1}}+V_{C}+R i_{2}$ and $V_{m}=-V_{D_{2}}-V_{C}+R i_{1}$, we obtain

$$
V_{D_{1}}+V_{D_{2}}+2 V_{C}+R\left(i_{2}-i_{1}\right)=0 \text {. }
$$

Because $\quad i_{2}-i_{1}=i_{2}-2 i_{1}+i_{1}=i_{m}-2 i_{1}$, and $-V_{c}=V_{D_{1}}+V_{D_{2}}$, from equation (13), we obtain

$$
i_{1}=\frac{V_{c}}{2 R}+\frac{i_{m}}{2} \text { and } i_{2}=-\frac{V_{c}}{2 R}+\frac{i_{m}}{2}
$$

Finally, equation (12) becomes

$$
V_{m}=\frac{1}{2}\left(R+R_{p}\right) i_{m}+\frac{1}{2 \alpha} \sinh ^{-1}\left(\frac{i_{m}}{2 I_{s}} e^{\alpha V_{c}+\alpha R_{p}\left(2 i_{c}+V_{c} / R\right)}\right) .
$$

Next, we focus on the state equation:

$$
\begin{aligned}
i_{c} & =C \frac{\mathrm{d} V_{c}}{\mathrm{~d} t} . \\
& =i_{D_{1}}-i_{1} \Longrightarrow C \frac{\mathrm{d} V_{c}}{\mathrm{~d} t}, \\
& =i_{D_{1}}-\frac{V_{c}}{2 R}-\frac{i_{m}}{2} .
\end{aligned}
$$

Now, to complete the state equation, we have to calculate $i_{D_{1}}$, which is straightforward (note that according to equation (2), $\mathbf{x}=V_{c}$ ):

$$
\begin{aligned}
\frac{V_{m}-V_{D_{1}}}{R} & =i_{1}, \\
& =\frac{V_{c}}{2 R}+\frac{i_{m}}{2} \Rightarrow V_{D_{1}}, \\
& =V_{m}-\frac{R i_{m}}{2}-\frac{V_{c}}{2} .
\end{aligned}
$$

Therefore, from equation

(16) and $i_{D_{1}}=I_{s}\left(e^{2 \alpha\left(V_{D_{1}}-R_{p} i_{D_{1}}\right)}-1\right)$, we obtain

$$
C \frac{\mathrm{d} V_{c}}{\mathrm{~d} t}=I_{s}\left(e^{2 \alpha V_{m}} e^{-\alpha R i_{m}} e^{-\alpha V_{c}} e^{-2 \alpha R_{p} i_{D_{1}}}-1\right)-\frac{i_{m}}{2}-\frac{V_{c}}{2 R} .
$$

By introducing equation (15), we obtain

$$
\begin{aligned}
C \frac{\mathrm{d} V_{c}}{\mathrm{~d} t}= & I_{s} e^{\sinh ^{-1}\left(i_{m} / 2 I_{s} e^{\alpha V_{c}+\alpha R_{p}\left(2 i_{c}+V_{c} / R\right)}\right)+\alpha R_{p} i_{m}-2 \alpha R_{p} i_{D_{1}}-\alpha V_{c}} \\
& -I_{s}-\frac{i_{m}}{2}-\frac{V_{c}}{2 R} .
\end{aligned}
$$

Because $i_{D_{1}}=i_{c}+\left(V_{c} / 2 R\right)+\left(i_{m} / 2\right)$, we obtain

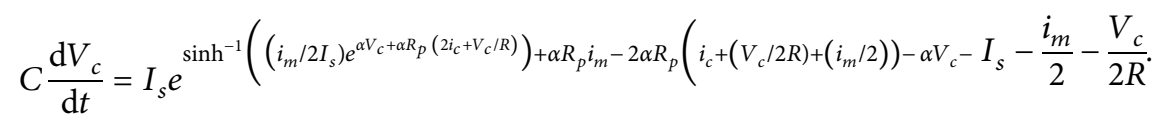


Finally, this circuit dynamic can be written as follows (where $V_{c o}$ is the initial capacitor value, i.e., the initially configured memory):

$$
\left\{\begin{array}{l}
V_{m}=\frac{1}{2}\left(R+R_{p}\right) i_{m}+\frac{1}{2 \alpha} \sinh ^{-1}\left(\frac{i_{m}}{2 I_{s}} e^{\alpha V_{c}+\alpha R_{p}\left(2 i_{c}+\left(V_{c} / R\right)\right)}\right), \\
C \frac{\mathrm{d} V_{c}}{\mathrm{~d} t}=I_{s} e^{\sinh ^{-1}\left(\left(i_{m} / 2 I_{s}\right) e^{\alpha V_{c}+\alpha R_{p}\left(2 i_{c}+\left(V_{c} / R\right)\right)}\right)-\alpha R_{p}\left(2 i_{c}+\left(V_{c} / R\right)\right)-\alpha V_{c}-I_{s}}-\frac{i_{m}}{2}-\frac{V_{c}}{2 R}, V_{c}(t=0)=V_{c o} .
\end{array}\right.
$$

From equation (21), one can see how the parasitic resistance $R_{p}$ affects the dynamic. In order to continue, we reasonably assume $R_{p} \approx 0$ because most diodes datasheets report $R_{p} \ll 1$.

According to equation (2), $V_{c}$ represents the inner state variable; however, it should be noted that the form $V_{m}=$ $R\left(V_{c}, i_{m}\right) i_{m}$ is not achieved (i.e., it does not contain $i_{m}$ proportionality). Instead, it can be naturally achieved through division by $i_{m}$ as follows:

$$
\left\{\begin{array}{l}
V_{m}=\left[\frac{1}{2} R+\frac{1}{2 \alpha i_{m}} \sinh ^{-1}\left(\frac{i_{m}}{2 I_{s}} e^{\alpha V_{c}}\right)\right] i_{m} \text { for } i_{m} \neq 0, \\
V_{m}=0 \text { for } i_{m}=0 \\
\frac{\mathrm{d} V_{c}}{\mathrm{~d} t}=\frac{I_{s}}{C}\left[e^{\left.\sinh ^{-1}\left(\left(i_{m} / 2 I_{s}\right) e^{\alpha V_{c}}\right)-\alpha V_{c}-1\right]-\frac{i_{m}}{2 C}-\frac{V_{c}}{2 R C}, V_{c}(t=0)=V_{c o}}\right.
\end{array}\right.
$$

where according to equation (2) the term $R\left(V_{c}, i_{m}\right)$ is

$$
\left\{\begin{array}{l}
R\left(V_{c}, i_{m}\right)=\frac{1}{2} R+\frac{1}{2 \alpha i_{m}} \sinh ^{-1}\left(\frac{i_{m}}{2 I_{s}} e^{\alpha V_{c}}\right) \text { for } i_{m} \neq 0, \\
R\left(V_{c}, i_{m}\right)=0 \text { for } i_{m}=0 .
\end{array}\right.
$$

Note that $R\left(V_{c}, i_{m}\right) \longrightarrow 0$ for $i_{m} \longrightarrow 0$; thus, it can be continuously extended to $i_{m}=0$ (the limit exists and can be obtained by L'Hospital's rule). Figure 2 shows in advance an example of the zero-crossing $V-i$ (the next section discusses the validation by simulation).

\section{Validation by Simulation}

The following parameters were used to simulate the memristor circuit emulator proposed in Figure 1: $R=270 \Omega$ and $C=0.5 \mu \mathrm{F}$. The assigned diode was $1 \mathrm{~N} 4148$ with a PSpicemodel card, model $1 \mathrm{~N} 4148 \mathrm{D}$ (Is $=2.52 \mathrm{n}, \mathrm{Rs}=0.568$, $N=1.752, \quad \mathrm{CJO}=4 \mathrm{p}, \quad M=0.4, \mathrm{TT}=20 \mathrm{n}, \quad$ Iave $=200 \mathrm{~m}$, $\mathrm{Vpk}=75, \mathrm{mfg}=$ OnSemi type $=$ silicon) [19]. The initial state condition $V_{c}=0$ was selected. The simulator used was LTspice [20]. For such diode models, the magnitudes of circuit parameters and the values of input signal amplitudes and frequencies are maintained similarly to compare the lobe shapes obtained in other works such as $[9,12,14,15]$. The current-voltage characteristics obtained from PSpice simulations are shown in Figures 2 and 3.

The following observations can be made from the simulation results. The loci in the $V$ - $i$ plane have hysteresis loops pinched at zero in the periodic steady state. The hysteresis loop shrinks to a single-valued function when the frequency increases and decreases, and the shape of the memristor depends on the circuit parameters and also retains the odd-symmetry property. The qualitative difference in the lobe shapes between the cases (a) and (b) of Figure 3 allows us to interpret that the dynamic of the memristor can exhibit a much richer behavior at low frequencies.

\section{Measurements, Experimental Setup, and Simulation}

The experimental setup is shown in Figure 4(b) and it comprises of two diodes model 1N4007, resistances $R=270 \Omega$, and the capacitor $C=0.5 \mu \mathrm{F}$. In order to test more case studies and to confirm that the current-controller emulator behavior is independent of the diode model, we use another diode type (which is the 1N4007) with respect to the above section.

We can see the emulator under test and also the oscilloscope-waveform generator Analog Discovery 2 [21]. The experimental result is shown in Figure 4(a) and the simulated result in Figure 5.

The excitatory signal is a sinusoidal waveform of frequency $f=1500 \mathrm{~Hz}$ and current amplitude of $I_{o}=0.74 \mathrm{~mA}$ (selected as similar as possible to that shown in [8], in order to compare the resulting $v-i$ characteristic). We use a voltage-controlled current source (VCCS), i.e., Howland current source (EHCS) circuits with a maximum output current of value of $1 \mathrm{~mA}$. This voltage-controlled current source was built with an OpAmp MCP6004 from Microchip Inc.

We conclude that the experimental measurement shows the same waveform pattern and the performance predicted by theory (Figure 4(a)) and by the emulator circuit simulations. Most important is to remark that the $i-v$ pattern is similar to one of the references [8] (for which the maximum current across the memristor is around $0.8 \mathrm{~mA}$ and the voltage $1 V$, with lobes in the $i-v$ pattern and a straight line 

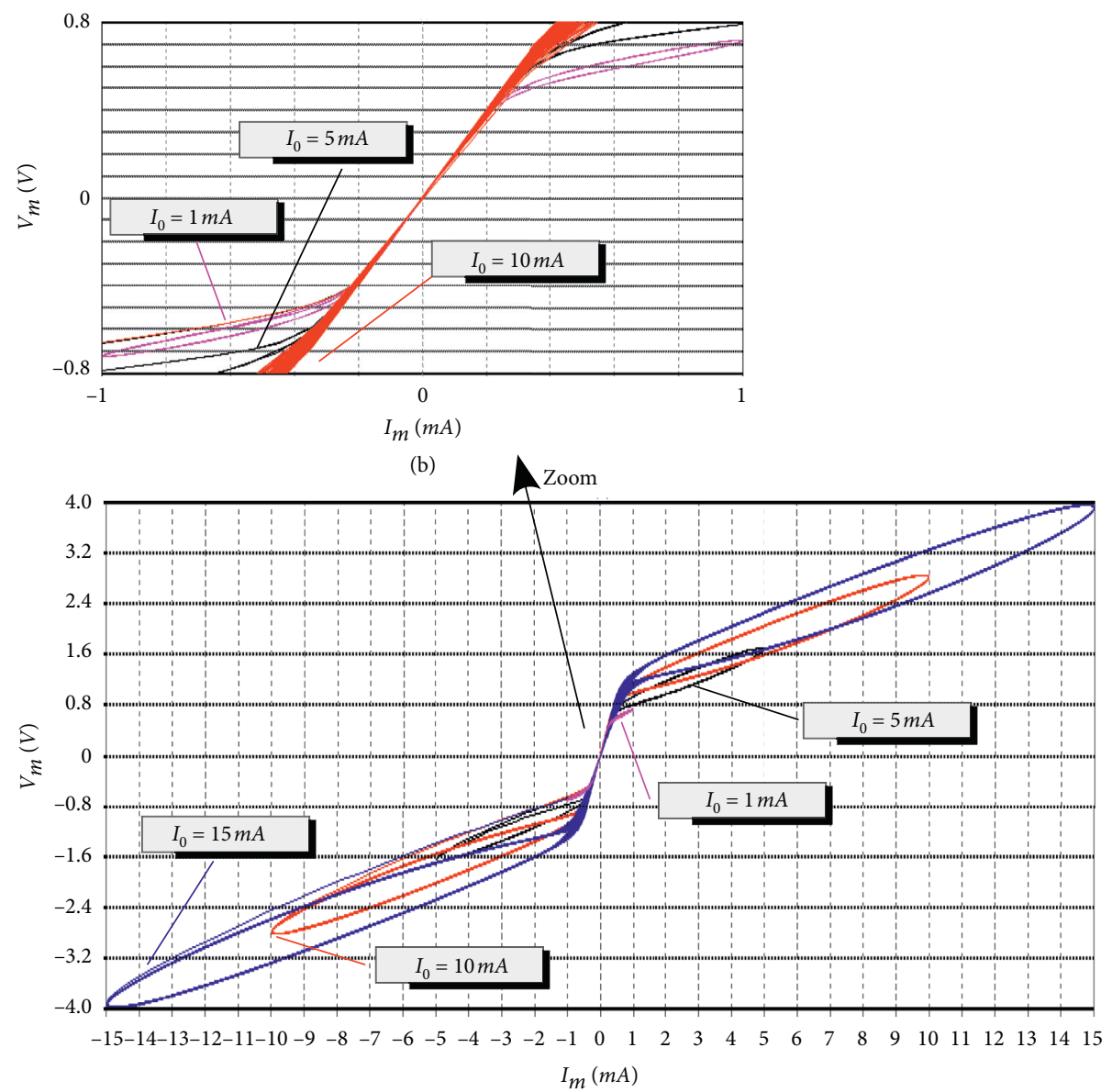

(a)

FIGURE 2: Current-voltage characteristics obtained through the LTspice simulations of the memristor emulator driven by a current source $i_{m}=I_{o} \sin (2 \pi f t)$ at a constant frequency $f=300 \mathrm{~Hz}$ with different $I_{o}$ values marked in $(\mathrm{a}): I_{o}=1 \mathrm{~mA}, I_{o}=5 \mathrm{~mA}, I_{o}=10 \mathrm{~mA}$, and $I_{o}=15 \mathrm{~mA}$. (b) An amplified view of the zero-crossing $V-i$ omitting the lobe at $I_{o}=15 \mathrm{~mA}$ to avoid the overwhelming superposition of curves.

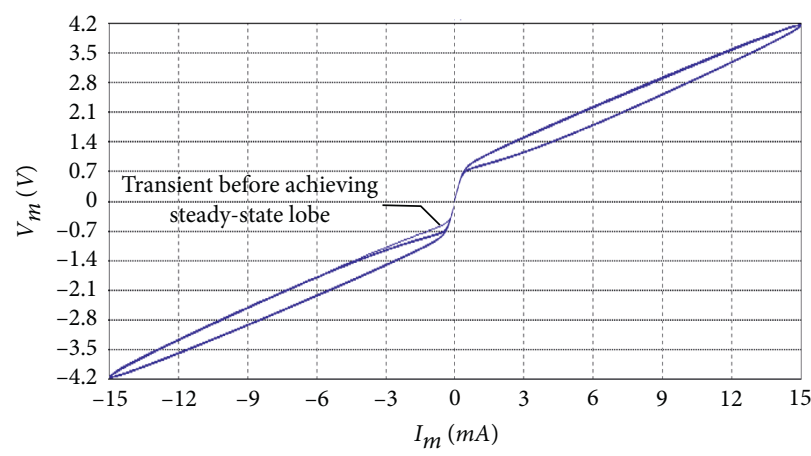

(a)

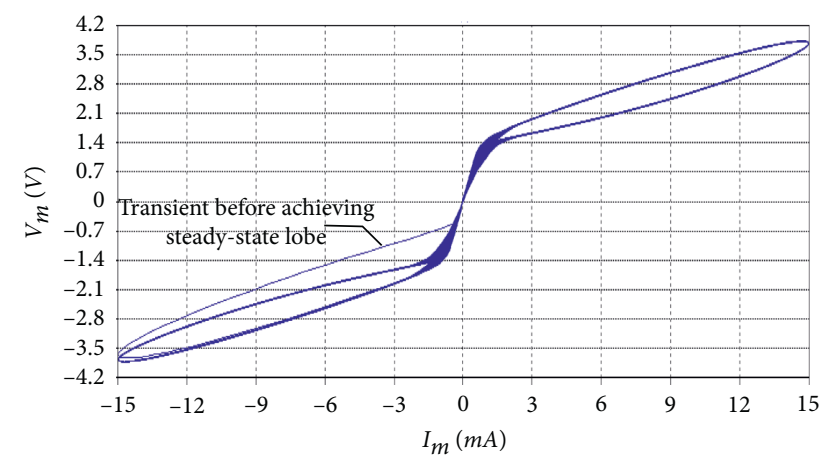

(b)

FIgURE 3: Continued. 


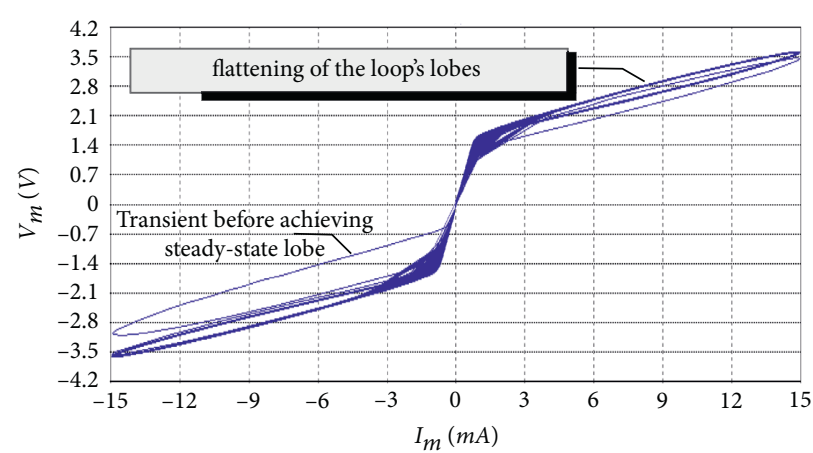

(c)

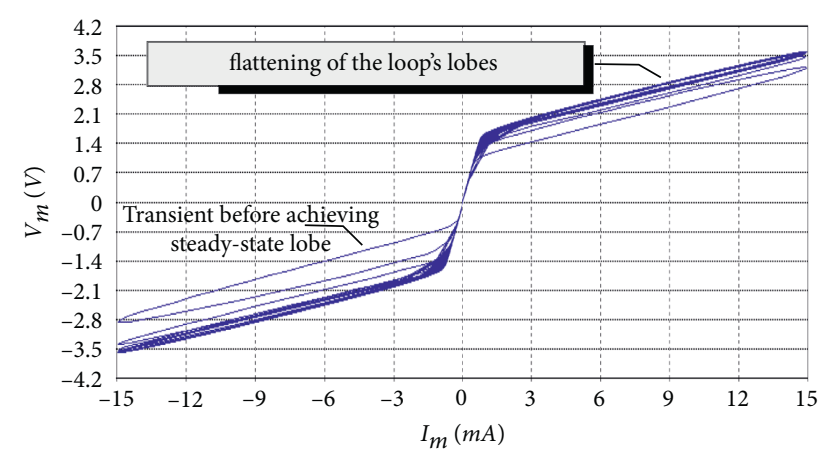

(d)

Figure 3: Simulated pinched hysteresis loop of the memristor emulator driven by a current source $i_{m}=I_{o} \sin (2 \pi f t)$ with a constant amplitude $I_{o}=15 \mathrm{~mA}$ at different $f$ values: (a) $f=100 \mathrm{~Hz}$, (b) $f=500 \mathrm{~Hz},(\mathrm{c}) f=1500 \mathrm{~Hz}$, and (d) $f=3000 \mathrm{~Hz}$ (100 cycles have been applied for each case study).

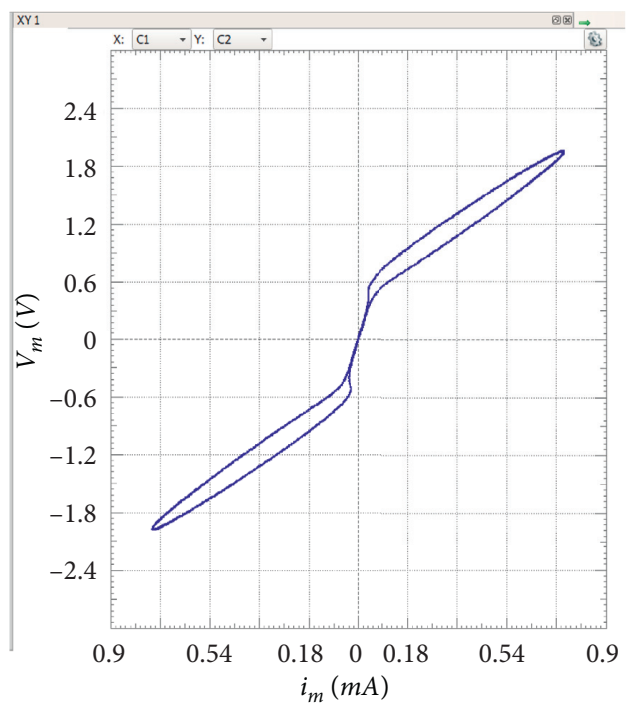

(a)

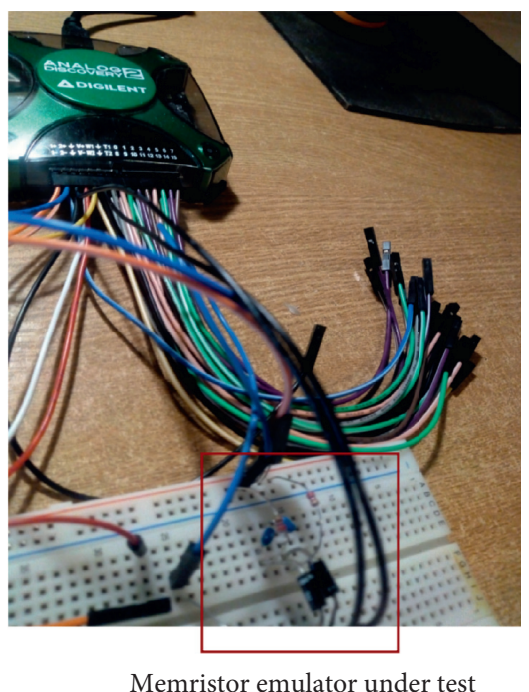

(b)

FIGURE 4: (a) $V$-i characteristics obtained through the measurements of an implemented memristor emulator driven by a current source $i_{m}=I_{o} \sin (2 \pi f t)$ at a constant frequency $f=1500 \mathrm{~Hz}$ with $I_{o}=0.74 \mathrm{~mA}$ and (b) view of the memristor emulator under test.

at zero), with the difference that such reference in the state of the art develops an active current-controlled memristor that needs a DC voltage power supply of $\pm 15 \mathrm{~V}$.

For completeness and comparison, the $v-i$ characteristics obtained through simulation of the measured case is shown in Figure 5, where is observed a minor difference in the slope at the origin that may be due to the diode model we use, which is model 1 N4007 D (Is $=7.02767 \mathrm{n}$, Rs $=0.0341512, \quad N=1.80803, \quad \mathrm{EG}=1.05743, \quad \mathrm{XTI}=5$, $\mathrm{BV}=1000, \mathrm{IBV}=5 e-08, \mathrm{CJO}=1 e-11, \mathrm{VJ}=0.7, \mathrm{M}=0.5$, $\mathrm{FC}=0.5, \mathrm{TT}=1 e-07, \mathrm{mfg}=\mathrm{OnSemi}$ type $=$ silicon $) \quad($ the diode model card has parasitic resistances and junction capacitance). Anyway, the experimental measurement shows the same waveform pattern and the performance predicted by theory and simulations, i.e., pinched hysteresis loop.

\section{Volatility Test for Neuromorphic Computing}

The emulator should not retain its value when no input signal is applied. The volatile memristor that features decay of device memory has high similarity to the biological synapses of neurons, and since neuromorphic computing is becoming more important, the decay is a desired feature.

At this point, it is important to underline that this kind of memristor emulator is not suitable for the logic-in-memory (LiM) paradigm, nor high-frequency applications; instead, our proposed circuit can be used practically for neuromorphic computing and as a candidate for mimicking biological synaptic functions. It is enough to show as example Figure 6 where an input current pulse train of $0.1 \mathrm{~mA}$ amplitude (5 cycles) is applied. In this case study, the 


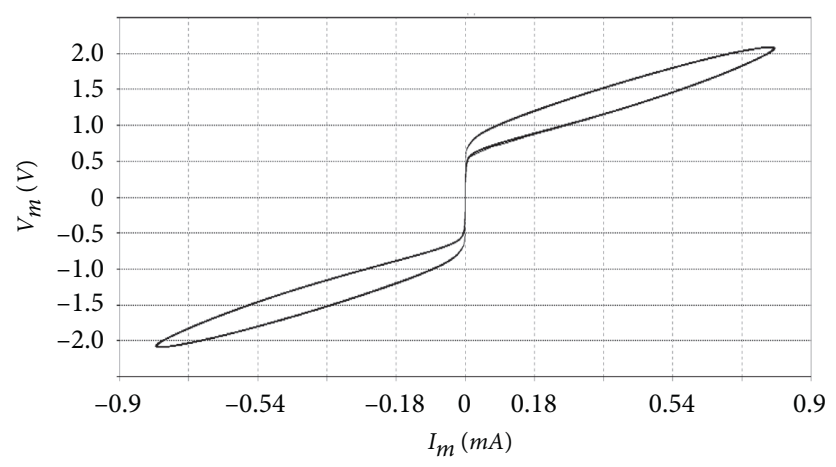

FIgURE 5: For completeness and comparison, the $V$ - $i$ characteristics obtained through simulation of the measured case (Figure 4).

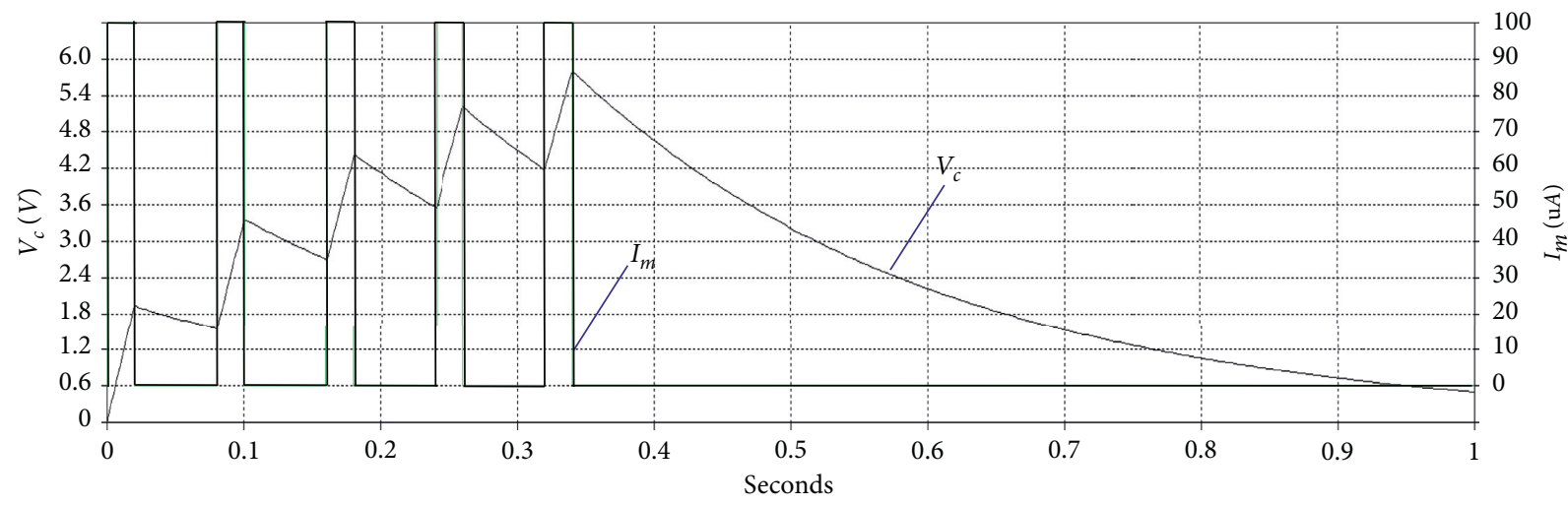

FIgURE 6: Variation of $V_{c}(t)$ (memory) with time for a given current input pulse.

emulator uses resistances $R_{1}=R_{2}=270 \mathrm{k} \Omega$ and the proposed emulator shows spontaneous decay or better said volatile nature similarity to the biological nervous system.

\section{Conclusion}

This work shows a passive circuit current-controlled memristor emulator. It overcomes the lack of currentcontrolled memristor commercial devices. Moreover, it covers a gap in the state of the art because, currently, only passive circuit voltage-controlled memristor emulators have been developed and used. The mathematical model of the proposed memristor emulator was derived and verified by simulations (the mathematical treatment was simplified and the diode parasitic capacitance was not taken into account because in that case the device dynamics should be studied with intensive numerical simulations (using for instance MATLAB) because the system is strongly nonlinear. Nevertheless, the SPICE simulator has the complete diode model with junction capacitance included; then, numerical circuital simulations are accurate). The circuit can be improved to be less symmetric in order to have a different clockwise and anticlockwise slope.

\section{Data Availability}

Simulation files link can be found at https://www.dropbox. com/s/q25pbrmlvsu1n1z/pspicefiles.zip?dl=0.

\section{Disclosure}

Part of this manuscript was submitted as a preprint in the link "https://arxiv.org/pdf/2008.08925.pdf".

\section{Conflicts of Interest}

The authors declare that they have no conflicts of interest.

\section{References}

[1] P. Mazumder, S. M. Kang, and R. Waser, "Memristors: devices, models, and applications [scanning the issue]," Proceedings of the IEEE, vol. 100, no. 6, pp. 1911-1919, 2012.

[2] S. Hamdioui, S. Kvatinsky, G. Cauwenberghs et al., "Memristor for computing: myth or reality?" in Proceedings of the Design, Automation and Test in Europe Conference and Exhibition (DATE), pp. 722-731, Lausanne, Switzerland, March 2017.

[3] M. Selmy, H. Mostafa, and A. Dessouki, "Low power memristor based voltage controlled oscillator for electrical neural stimulation," in IEEE International Conference on Advanced Control Circuits and Systems and New Paradigms in Electronics and Information Technology, pp. 344-347, Hong Kong, China, May 2017.

[4] B. Ramakrishnan, A. Durdu, K. Rajagopal, and A. Akgul, "Infinite attractors in a chaotic circuit with exponential memristor and josephson junction resonator," AEU-International Journal of Electronics and Communications, vol. 123, Article ID 153319, 2020. 
[5] P. Khurana, K. Singh, and A. Sharma, "A hybrid cmosmemristor based programmable wien bridge oscillator," in Proceedings of the 3rd IEEE International Conference on Recent Trends in Electronics, Information and Communication Technology, RTEICT, Bangalore, India, May 2018.

[6] M. Zidan, H. Omran, C. Smith, A. Syed, A. Radwan, and K. Salama, "A family of memristor-based reactance-less oscillators," Engineering," International Journal of Circuit Theory and Applications, vol. 42, no. 11, pp. 1103-1122, 2013.

[7] A. Mosad, M. Fouda, M. Khatib, K. Salama, and A. Radwan, "Improved memristor-based relaxation oscillator," Microelectronics Journal, Elseiver, vol. 44, no. 9, 2013.

[8] G. Abdullah, J. Zainulabideen, M. Fouda, and M. H. Chowdhury, "A new simple emulator circuit for current controlled memristor," in Proceedings of the 2015 IEEE International Conference on Electronics, Circuits, and Systems (ICECS), pp. 288-291, Cairo, Egypt, December 2015.

[9] F. Corinto and A. Ascoli, "Memristive diode bridge with LCR filter," Electronics Letters, vol. 48, no. 14, pp. 824-825, 2012.

[10] M. Chen, M. Li, Q. Yu, B. Bao, Q. Xu, and J. Wang, "Dynamics of self-excited attractors and hidden attractors in generalized memristor-based Chua's circuit," Nonlinear Dynamics, vol. 81, no. 1-2, pp. 215-226, 2015.

[11] Q. Xu, N. Wang, B. Bao, M. Chen, and C. Li, "A feasible memristive chua circuit via bridging a generalized memristor," Journal of Applied Analysis and Computation, vol. 6, no. 4, pp. 1152-1163, 2015.

[12] M. Chen, J. Yu, Q. Yu, C. Li, and B. Bao, "A memristive diode bridge-based canonical chua's circuit," Entropy, vol. 16, no. 12, pp. 6464-6476, 2014.

[13] Z. T. Njitacke, J. kengne, H. B. Fotsin, A. N. Negou, and D. Tchiotsop, "Coexistence of multiple attractors and crisis route to chaos in a novel memristive diode bidge-based jerk circuit," Chaos, Solitons \& Fractals, vol. 91, pp. 180-197, 2016.

[14] Q. Xu, Q. Zhang, N. Wang, H. Wu, and B. Bao, "An improved memristive diode bridge-based band pass filter chaotic circuit," Mathematical Problems in Engineering, vol. 201711 pages, 2017.

[15] B. C. Bao, P. Y. Wu, H. Bao, H. G. Wu, X. Zhang, and M. Chen, "Symmetric periodic bursting behavior and bifurcation mechanism in a third-order memristive diode bridgebased oscillator," Chaos, Solitons \& Fractals, vol. 109, pp. 146-153, 2018.

[16] A. Yesil, "A new grounded memristor emulator based on mosfet-c," AEU-International Journal of Electronics and Communications, vol. 91, 2018.

[17] L. Zhijun, Z. Yicheng, and M. Minglin, "A novel floating memristor emulator with minimal components," Active and Passive Electronic Components, vol. 2017, 2017.

[18] A. Alharbi and M. Chowdhury, "Simple current-controlled memristor emulators," in Memristor Emulator CircuitsSpringer, Berlin, Germany, 2020.

[19] LTwiki, "Standard.dio," 2019, http://ltwiki.org/index.php? title $=$ Standard.dio.

[20] A Devices, "Spice Simulation Software," 2019, http://www. analog.com/en/design-center/design-tools-and-calculators.

[21] “analog Discovery 2,” 2020, https://www.reference. digilentinc.com/. 